\section{Avaliação da rede de atenção ao portador de hipertensão arterial: estudo de uma região de saúde}

\author{
Evaluation of the healthcare network for persons \\ with arterial hypertension: study of a \\ health district
}

\section{Evaluación de la red de atención al paciente de hipertensión arterial: estudio de una región de salud}

\section{Resumo}

No contexto das politicas públicas de saúde, a rede de atenção é uma estratégia que visa a promover a equidade do acesso das pessoas aos serviços e reduzir a sua fragmentação. O objetivo foi avaliar o grau de desenvolvimento dos componentes de uma rede de atenção à saúde para hipertensão. Trata-se de um estudo avaliativo do tipo ex-ante, de corte transversal, voltado à implantação da Rede de Atenção à Saúde das pessoas com doenças crônicas com a aplicação de questionário a 17 gestores de saúde dos municípios que formam a maior região de saúde de Mato Grosso do Sul, Brasil. O questionário é constituído de 65 questões que contemplam os cinco componentes: Atenção Primária à Saúde; Atenção Especializada; Sistemas de Apoio; Sistemas Logísticos; e Governança. Foram realizados testes estatísticos descritivos e a classificação dos serviços prestados em cada componente por meio do teste de Friedman, seguido pelo pós-teste de Student-Newman-Keuls, com nível de significância de 5\%. Os resultados foram distribuídos em quartis e apresentados em gráficos Boxplot. Foram estabelecidas correlações entre as dimensões. Resultados apontam que os componentes estão no grau intermediário de implantação, com baixo desenvolvimento nos quesitos necessários para a constituição de redes. A Atenção Primária à Saúde não coordena o cuidado, e os componentes Atenção Especializada e Governança apresentaram os piores resultados. Os achados apontam predomínio de serviços instalados ainda distantes das práticas necessárias para a composição de redes de atenção à saúde, podendo comprometer a sua implantação.

Política de Saúde; Serviços de Saúde; Avaliação em Saúde; Hipertensão
Cleuzieli Moraes dos Santos 1

Ana Rita Barbieri 2

Crhistinne Cavalheiro Maymone Gonçalves 3

Daniel Henrique Tsuha 1

doi: 10.1590/0102-311X00052816

\author{
Correspondência \\ C. M. Santos \\ Rua 14 de Julho 5093, Residencial Indaiá, apto. 14, Bloco C, \\ Campo Grande, MS 79011-470, Brasil. \\ enf_cleuzieli@hotmail.com \\ 1 Secretaria de Estado de Saúde de Mato Grosso do Sul, Campo \\ Grande, Brasil. \\ 2 Universidade Federal do Mato Grosso do Sul, Campo Grande, \\ Brasil. \\ 3 Universidade Federal da Grande Dourados, Dourados, Brasil.
}




\section{Introdução}

O Brasil apresenta mudanças na sua estrutura etária com o aumento no percentual de idosos e exibe alterações no cenário epidemiológico com uma "tripla carga de doenças", pela presença concomitante das doenças infecciosas e carenciais, causas externas e doenças crônicas, além das alterações nutricionais 1 . No cenário das doenças crônicas, a hipertensão arterial alcança prevalência de 21,4\% entre adultos, alcançando 55\% das pessoas acima de 75 anos 2. Em 2012, de 14.634 óbitos ocorridos em Mato Grosso do Sul, 4.311 (29,4\%) foram decorrentes das doenças do aparelho circulatório, sendo 739 (5\%) causados por doenças hipertensivas, proporção superior a do país (28,2\% e 3,8\%) e da Região Centro-oeste (27,3\% e 3,8\%), respectivamente (Departamento de Informática do Sistema Único de Saúde. Informações de saúde: estatísticas vitais. Mortalidade. 2012. http://www2.datasus.gov.br/ DATASUS/index.php?area=01, acessado em 16/Jul/2014).

Nesse cenário, evidências internacionais apontam melhores resultados sanitários e econômicos nas regiões que organizam os seus sistemas de saúde em redes de atenção ${ }^{3}$. Essa conformação tem sido a resposta eficaz para a superação da fragmentação do cuidado e da crise dos sistemas de atenção à saúde 3,4. Silva 5 e Magalhães Júnior 6 descrevem que redes de atenção favorecem a oferta de ações integrais centradas no usuário e asseguram a otimização da oferta assistencial e dos recursos, conferindo eficácia ao sistema de saúde. Para o momento sanitário atual, no qual se buscam alternativas para o alcance da equidade no acesso aos serviços de saúde de maior complexidade, essa estruturação pode ser considerada a melhor estratégia para o enfrentamento dos problemas de saúde pública 5,6 .

Redes de atenção estão na agenda e no arcabouço legal da política de saúde brasileira há mais de três décadas e, mais incisivamente, a partir da Portaria no 4.279/2010 7 e do Decreto no 7.508/2011 8 que estabeleceram um eixo regulatório 4 . Após a municipalização da gestão dos sistemas de saúde nos anos 1990, nos anos 2000 foi necessária a adoção de medidas que propiciassem a organização do sistema de saúde por regiões. A publicação da Norma Operacional da Assistência à Saúde (NOAS) em 2001, valorizando a regionalização, visava a superar a fragmentação do cuidado e as desigualdades regionais aprofundadas com a transferência da gestão da saúde aos municípios 9,10.

Em 2010, o Ministério da Saúde divulgou as diretrizes para a conformação da Rede de Atenção à Saúde (RAS) como uma forma de organizar o sistema de saúde nos âmbitos local e regional, considerando as diferentes densidades e complexidades tecnológicas em um território definido. As RAS são constituídas de cinco componentes: (1) Atenção Primária à Saúde; (2) Atenção Especializada; (3) Sistemas de Apoio; (4) Sistemas Logísticos; e (5) Governança 6,11. O entendimento operacional dos componentes é fundamental para o sucesso da implantação das RAS no que tange à equidade no acesso e à otimização de ações e serviços frente às necessidades de saúde e ao perfil epidemiológico dos usuários 12 .

Com o Plano de Ações Estratégicas para o Enfrentamento das Doenças Crônicas não Transmissíveis, para o período de 2011 a 2022, foi proposta a formação da Rede de Atenção à Saúde das Pessoas com Doenças Crônicas, organizada por meio de linhas de cuidado prioritárias (doenças renocardiovasculares - hipertensão arterial e insuficiência renal crônica - diabetes, obesidade, doenças respiratórias crônicas e câncer) permitindo, assim, tanto a organização dos serviços quanto a delimitação da linha de cuidado, com o fluxo a ser percorrido pelos usuários 1 .

A implantação da Rede de Atenção à Saúde das Pessoas com Doenças Crônicas poderá dar respostas importantes para a assistência e, em especial, para a hipertensão arterial, que apresenta quadros elevados de morbidade e mortalidade. Por isso, é relevante avaliar o contexto organizacional e epidemiológico antes da sua instalação 13 .

Assim, compreendendo que as redes constituem uma política pública para atender ao princípio da equidade no acesso das pessoas aos serviços de saúde, e dada a exiguidade de instrumentos para avaliar a sua estrutura e organização, este artigo tem como objetivo apresentar os resultados da avaliação do grau de desenvolvimento dos componentes de uma rede de atenção a portadores de hipertensão arterial na região de saúde de Campo Grande, Mato Grosso do Sul, Brasil, para a estruturação da Rede de Atenção à Saúde das Pessoas com Doenças Crônicas. 


\section{Método}

\section{Tipo de estudo}

Foi realizado um estudo transversal que avaliou ex-ante o desenvolvimento dos componentes da Rede de Atenção à Saúde das Pessoas com Doenças Crônicas, em particular com enfoque na rede de atenção à hipertensão, porque, considerando sua magnitude, há protocolos consolidados para todas as instâncias assistenciais e manejo disseminado para o agravo. Esse tipo de avaliação permite a análise da estrutura e dos processos de trabalho antes da efetivação de um programa ou de uma ação. É possível emitir julgamentos acerca da relevância ou da viabilidade da estratégia selecionada para o enfrentamento da situação-problema, permitindo, assim, ordenar recursos e ações segundo a sua eficiência 14,15 . Outro aspecto a ser considerado é o contexto em que ocorrerá a implantação, que tanto modifica o modo como ocorre uma intervenção quanto contribui diretamente para o seu processo, o que suscita, ainda mais, a necessidade de conhecer a realidade na qual novas intervenções estão sendo propostas 13 .

\section{Área do estudo}

A pesquisa foi realizada na Região de Saúde de Campo Grande, composta por 17 municípios: Bandeirantes, Camapuã, Campo Grande, Chapadão do Sul, Costa Rica, Corguinho, Figueirão, Jaraguari, Maracaju, Nova Alvorada do Sul, Paraíso das Águas, Ribas do Rio Pardo, Rio Negro, Rochedo, São Gabriel do Oeste, Sidrolândia e Terenos. Conta com 1.056.932 habitantes (trata-se da região mais populosa), concentra $42,19 \%$ da população do estado e os serviços ambulatoriais e hospitalares de maior complexidade, sendo Campo Grande a capital.

\section{Instrumento e participantes do estudo}

Foi elaborado um questionário adaptado baseando-se nos instrumentos propostos por Mendes $16 \mathrm{e}$ Chueiri 17. Para a sua formulação foram considerados os componentes previstos para a RAS, sem a análise das especificidades das normas previstas para a rede de doenças crônicas não transmissíveis e hipertensão arterial. A orientação para o seu preenchimento indicava que as respostas deveriam ter como base as ações previstas para a linha de cuidado a ser implantada para a hipertensão arterial.

O questionário elaborado considerou os cinco componentes previstos para uma RAS com 65 questões, sendo distribuídas 15 para o componente Atenção Primária à Saúde, que incluiu o uso do Programa Telessaúde, não contemplado nos instrumentos propostos pelos autores; sete para Atenção Especializada (ambulatórios, hospitais e serviços de urgência e emergência); 13 para o componente Sistema de Apoio (sistema de apoio diagnóstico e terapêutico, assistência farmacêutica e sistema de informação em saúde); dez para Sistemas Logísticos (cartão de identificação, prontuário clínico, regulação e sistema de transporte); e vinte para Sistema de Governança.

O questionário foi testado previamente por um secretário de saúde municipal não pertencente à região de saúde estudada, que não referiu dificuldades e o definiu como de fácil compreensão e preenchimento.

Os 17 gestores municipais convidados aceitaram participar do estudo. O questionário lhes foi entregue e eles foram orientados quanto ao preenchimento, considerando que deveriam responder às questões conforme sua compreensão do grau de desenvolvimento de cada item, considerada a linha de cuidado para hipertensão arterial.

A distribuição ocorreu nas reuniões da Comissão Intergestores Bipartite no período de novembro de 2014 a março de 2015. A devolução foi feita entre novembro de 2014 e agosto de 2015, com 100\% de questionários respondidos.

Os itens do questionário tinham respostas dentro de um escala do tipo Likert de quatro categorias: 0 - inexistente; 1 - incipiente; 2 - insuficiente; 3 - ótimo. A categoria 0 demonstra a não existência do item ; a 1 expressa a existência, mas com um funcionamento básico ou incipiente, sem normas e protocolos instituídos; 2 aponta a existência com funcionamento normatizado e razoavelmente bom, 
mas ainda insuficiente quanto ao uso de protocolos ou cobertura do serviço; e a categoria 3 indica existência em operação, de forma ótima, considerando a normalização e cobertura.

O escore de cada atributo foi definido pela somatória das respostas e dividido pelo total de municípios participantes. Foram calculadas as medianas e os intervalos interquartis e valores discrepantes com apresentação dos resultados em gráficos do tipo Boxplot 18. A análise estatística foi realizada por meio do programa SigmaPlot (Systat Software, San Jose, Estados Unidos), versão 12.5, considerando um nível de significância de $5 \%$.

Este estudo, recorte da pesquisa de mestrado profissional em saúde da família, foi aprovado pelo Comitê de Ética em Pesquisa da Universidade Federal de Mato Grosso do Sul (CONEP 466/2012), com o protocolo número 773.454, e recebeu financiamento da Fundação de Apoio ao Desenvolvimento do Ensino, Ciência e Tecnologia do Estado do Mato Grosso do Sul/Conselho Nacional de Desenvolvimento Científico e Tecnológico (FUNDECT/CNPq no 06/2011 - PRONEM).

\section{Resultados}

Em relação ao componente da Atenção Primária à Saúde, observa-se que os municípios contam com bom desenvolvimento na definição do território e da população adscrita, cadastro das famílias e indivíduos, e definição dos limites geográficos (Figura 1).

A Figura 1 demonstra a dificuldade para estabelecer um fluxo contínuo de comunicação entre os serviços de diferentes complexidades da rede para a abordagem da situação de saúde dos indivíduos, excetuando-se as situações de urgência e emergência que informam sobre o atendimento. Os resultados apontam problemas para agendar os pacientes com outros níveis de complexidade e, também, falta de contrarreferência.

A Atenção Especializada é o componente menos desenvolvido nos municípios (Figura 2). Chama atenção a falta de integração dos serviços especializados com os demais serviços. Os municípios não conhecem seu território, não desenvolvem programação das atividades voltadas às necessidades do território e não estabelecem mecanismos de contrarreferência para a Atenção Primária à Saúde.

Há distribuição de pontos de coleta de exames bioquímicos, bem como a de insumos farmacêuticos e medicamentos, situação coerente com as necessidades de saúde da população dos territórios.

Constatou-se desenvolvimento incipiente do sistema de apoio diagnóstico e de imagem baseado na economia de escala, necessidades de saúde da população e indicação de exames de imagem (Figura 3).

Em grande parte dos municípios não há base de dados única da população usuária, mesmo com o cartão SUS (Sistema Único de Saúde) implantado. Não há prontuário eletrônico em todos os pontos de atenção. Apesar de instituído o serviço de regulação para serviços de emergência e urgência, não há médicos para regular o serviço em mais da metade dos municípios.

Existem formas instituídas de transporte de pessoas em situação de urgência e emergência para procedimentos eletivos e transporte de resíduos (Figura 4).

A Governança esteve presente nas categorias inexistente e incipiente (Figura 5). Embora exista um sistema de contratação de serviços, este não está alinhado à RAS. As respostas dos gestores para os itens voltados à Governança evidenciam que não está institucionalizado o processo de monitoramento e avaliação. Ações como educação permanente também são incipientes para grande parte dos municípios e, quando existem, não estão voltadas para a implantação de novos modelos de atenção à saúde.

\section{Discussão}

A pesquisa evidencia a presença dos componentes necessários à implantação da rede de atenção a portadores de hipertensão arterial nos municípios estudados. No entanto, observa-se heterogeneidade no grau do seu desenvolvimento, com melhores resultados na Atenção Primária à Saúde e Estratégia Saúde da Família. A participação dos serviços especializados e o apoio diagnóstico, com resultados inexistentes ou incipientes, precisam ser incluídos na organização da rede. A Governança é pouco desenvolvida para a racionalidade da RAS, cujo êxito depende da articulação e comprometimento entre as diferentes instituições e gestores. 
Figura 1

Distribuição de respostas do componente Atenção Primária à Saúde. Região de Saúde de Campo Grande, Mato Grosso do Sul, Brasil, $2014-2015$.

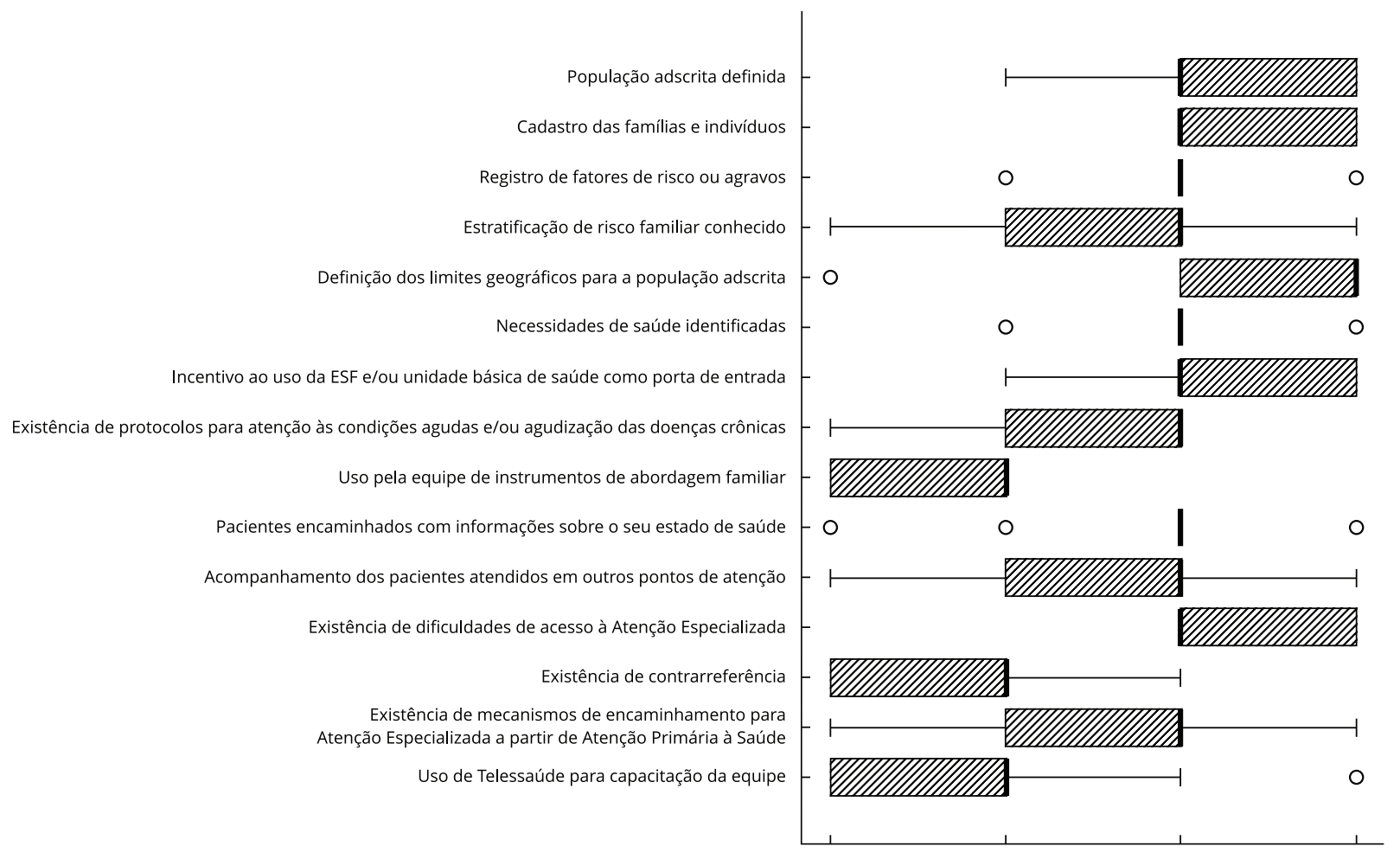

ESF: Estratégia Saúde da Família.

Para a operacionalização adequada dos sistemas de saúde em resposta às necessidades da população, é imprescindível a coordenação da Atenção Primária Saúde com o envolvimento de outros pontos de atenção, considerando os recursos humanos, financiamento, informações, comunicação e transporte. A comunicação entre os pontos de atenção deve acontecer baseada em relações horizontalizadas, que promovam a reorganização dos serviços em outra lógica. É com base na reestruturação dos serviços que se viabiliza a consolidação das redes assistenciais 19.

No entanto, as respostas deste estudo sugerem que a integralidade do cuidado é incipiente em decorrência das fragilidades no acesso e na comunicação entre os pontos de atenção. De forma geral, as ações voltadas para a gestão do cuidado às famílias com o uso de ferramentas para uma abordagem integral não é uma prática difundida, tanto pela insuficiente utilização de ferramentas como pela insuficiência/incipiência da utilização de protocolos para atenção às condições agudas e/ou agudização das doenças crônicas.

A conformação em RAS, tendência internacional, visa a superar o cuidado fragmentado, os custos elevados, com expectativa de maior eficiência ao sistema 20,21,22. Quando baseada em território, a RAS reduz as desigualdades locorregionais e as iniquidades de acesso ${ }^{9}$. O uso do território como base para a organização do serviço aproxima os sujeitos e suas coletividades, a partir de uma identificação de necessidades singulares e estabelecimentos de relações com vínculo e acolhimento, preditores de cenários de integralidade do cuidado, princípio doutrinário da conformação dos serviços em redes.

$\mathrm{Na}$ Espanha, mesmo com a organização dos serviços em redes de atenção 23 , semelhante ao cenário encontrado no Brasil, o reconhecimento do processo de trabalho da Atenção Primária à 
Figura 2

Distribuição de respostas do componente Atenção Especializada. Região de Saúde de Campo Grande, Mato Grosso do Sul, Brasil, $2014-2015$.

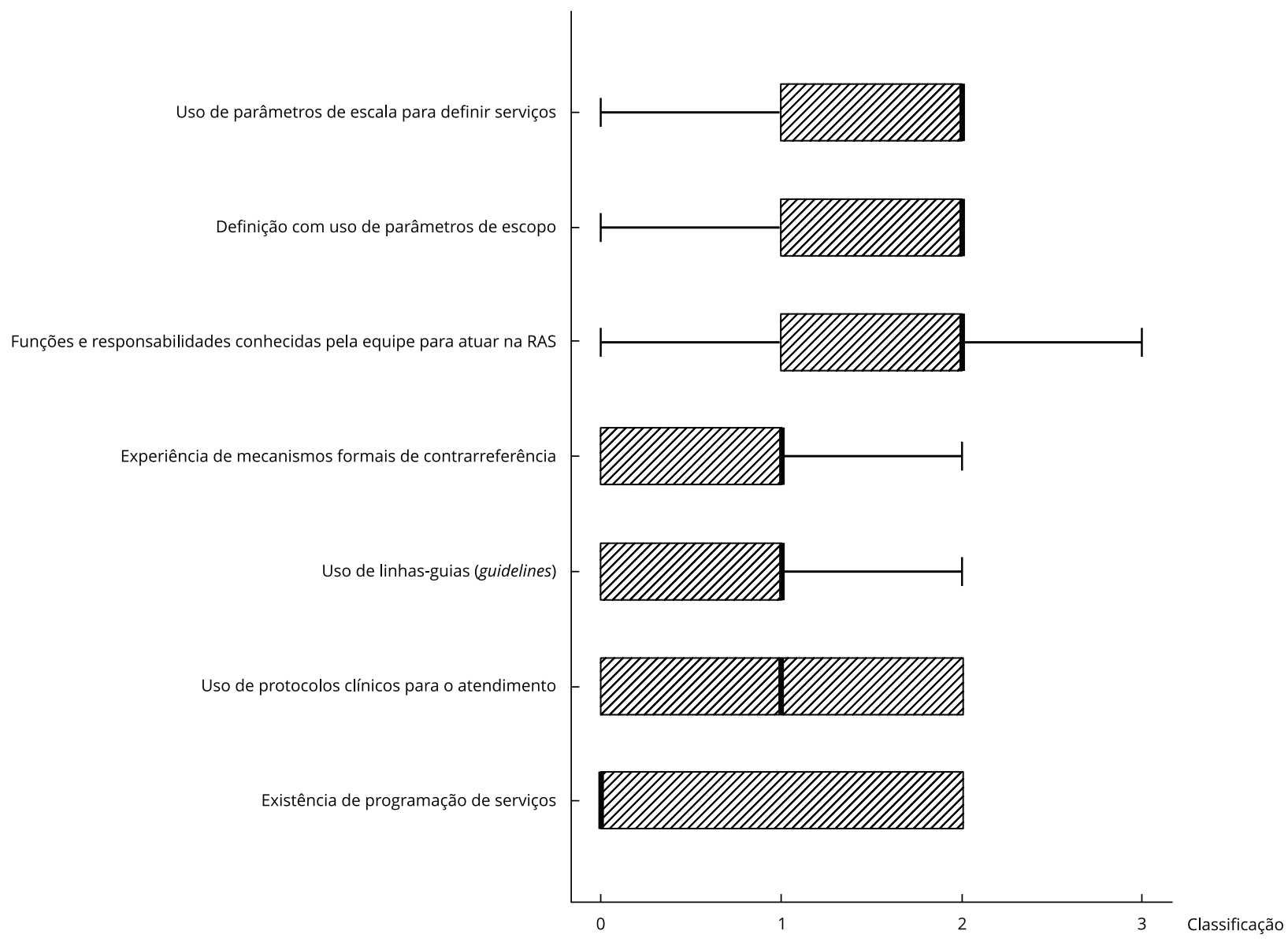

RAS: Rede de Atenção à Saúde.

Saúde pelos profissionais da atenção especializada é insuficiente 24. Problema também constatado em nosso estudo, em que se evidenciou que a atenção especializada não reconhece as suas atribuições e responsabilidades na rede, especialmente quanto à integração, comunicação e resolubilidade, fluxos e instrumentos de contrarreferência à Atenção Primária à Saúde. Os mecanismos de referência e contrarreferência são elementos fundamentais e resultam em condutas eficientes 19,22.

A integração entre serviços de densidades tecnológicas diversas é o desafio a ser perseguido pelas equipes de saúde, e uma ferramenta de apoio que pode ser explorada para esta aproximação é o Telessaúde, iniciativa de apoio e educação permanente, que é usada por apenas um município dentre os 17 analisados. Para o Estado de Mato Grosso do Sul, que tem um território extenso constituído por municípios de pequeno porte, esse Programa pode ser de grande valia para o apoio matricial visando à maior eficiência dos serviços e integração dos diferentes componentes da RAS.

Com resultados semelhantes aos deste estudo, avaliações de qualidade e pesquisas em diferentes territórios brasileiros identificaram fragilidades nos processos de trabalho que precisam ser enfrentadas, principalmente no que diz respeito ao uso de sistemas de informações, protocolos clínicos e de encaminhamento aos serviços especializados, coordenação e integração da rede 20,25,26. 


\section{Figura 3}

Distribuição de respostas do componente Sistema de Apoio. Região de Saúde de Campo Grande, Mato Grosso do Sul, Brasil, 2014-2015.

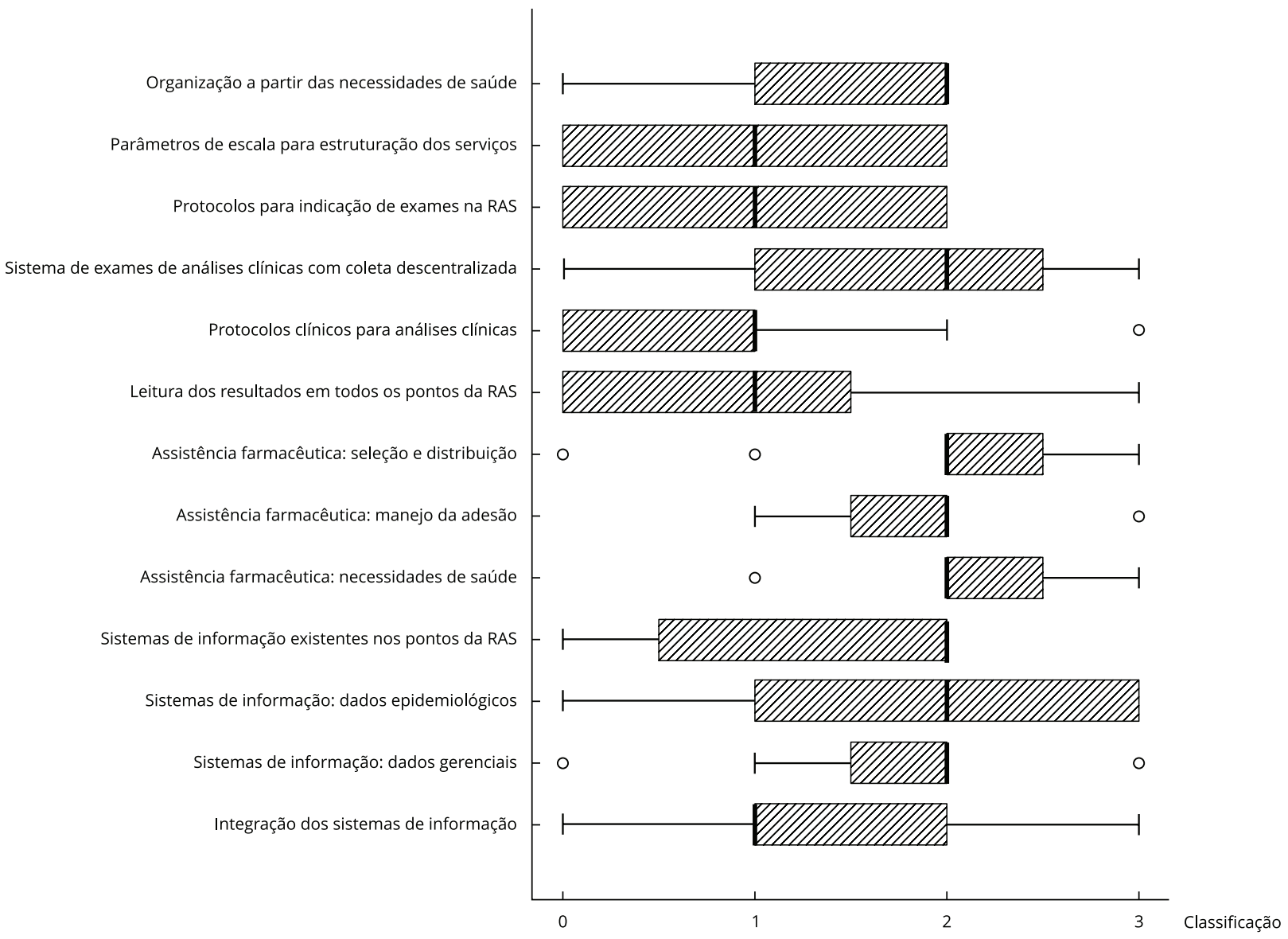

RAS: Rede de Atenção à Saúde.

Corroborando os achados desta pesquisa, outros estudos apontam que o acesso aos serviços especializados tem sido dificultado pela distância dos estabelecimentos de saúde, inadequado sistema de transporte sanitário e de comunicação entre os diversos pontos de atenção da rede 27,28. Também, os resultados demonstram que as diretrizes clínicas preconizadas para o aperfeiçoamento da assistência não são utilizadas nos serviços especializados, podendo criar situações de risco à segurança dos pacientes em atendimento e comprometer o itinerário e o tratamento. $\mathrm{O}$ cuidado às pessoas com doenças crônicas demanda a organização dos serviços mais robustos e integrados, de modo a garantir uma estreita integração entre a Atenção Primária à Saúde e os serviços especializados, proporcionando a condução ágil e oportuna dos usuários, só possível com qualidade técnica, fluxos para acesso aos serviços e financiamento compatível 22.

Apesar dos limites, é possível encontrar experiências que demonstram que os modelos de coordenação do cuidado por meio da integração e continuidade da informação entre os pontos de atenção não é uma utopia, pois $87 \%$ dos médicos generalistas no Reino Unido referenciam os seus pacientes com relatório constando as informações relevantes no segmento dos pacientes, realidade não muito diferente da Holanda e da França 20. 
Figura 4

Distribuição de respostas para o componente Sistema Logístico. Região de Saúde de Campo Grande, Mato Grosso do Sul, Brasil, 2014-2015.

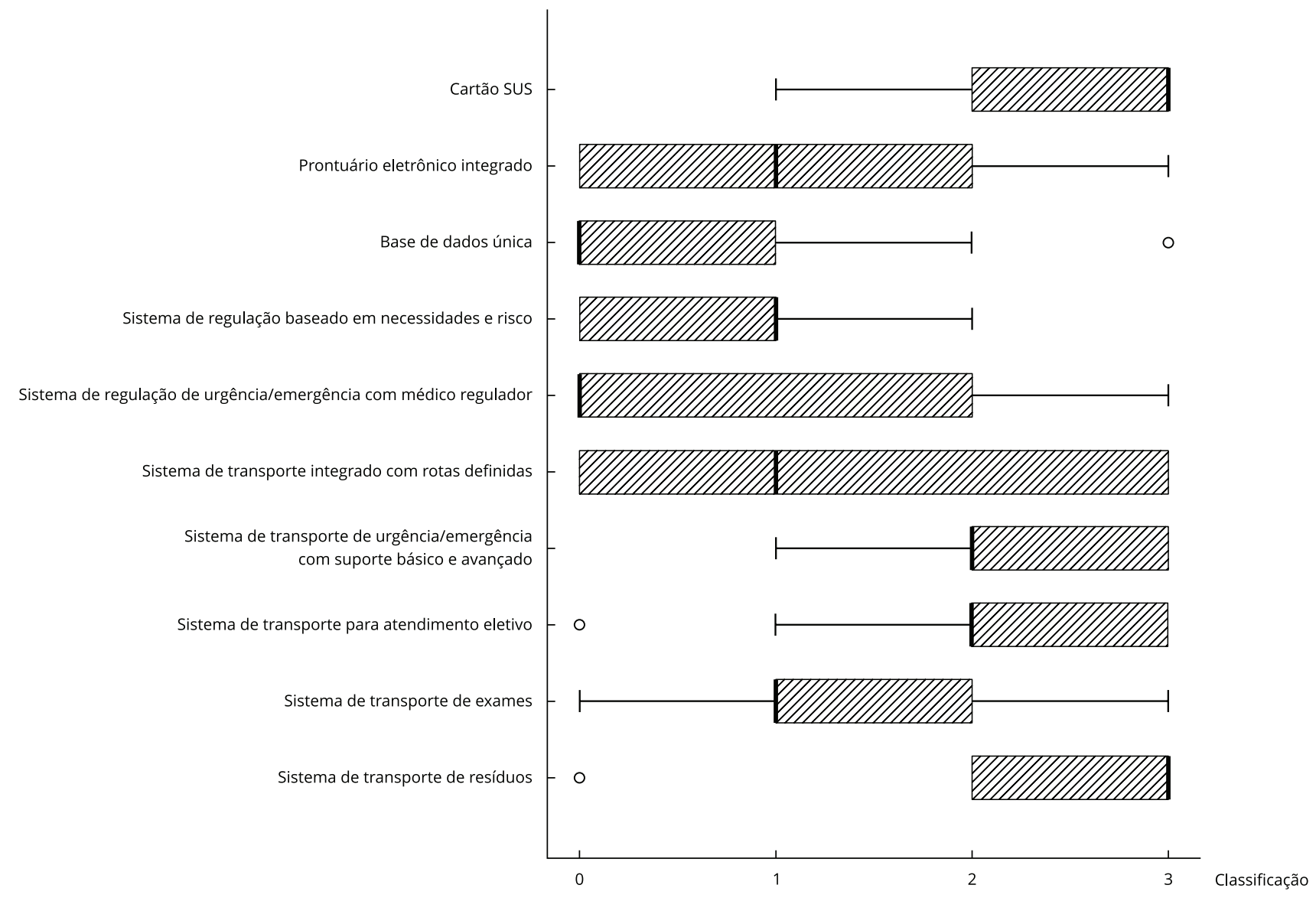

No Brasil, pesquisas demonstraram que a organização do sistema de saúde integrada é viável, pois parte do acesso aos serviços especializados é demandada pela Atenção Primária à Saúde por meio de serviços de regulação 29,30 .

Compreendendo que a atenção especializada deve obedecer ao conceito de economia de escala, no Brasil a oferta desses serviços tem distribuição heterogênea e eles estão desarticulados com os demais componentes da rede 27,31. Essa situação também se verifica em Mato Grosso do Sul, pois este estudo revelou que a coordenação dos serviços não atende aos preceitos dos parâmetros de escala e escopo, além do reconhecimento de papel e formas de organização na rede. Especificamente neste estado, as distâncias territoriais são expressivas e há vazios e lacunas assistenciais entre municípios e regiões que têm baixa densidade populacional. Portanto, identificar as necessidades de saúde e buscar estratégias que respeitem a especificidade dos territórios implica o uso da economia de escala, sem ferir a equidade no acesso das pessoas aos serviços que são necessários.

A Atenção Especializada, caracterizada pela exígua disponibilidade e demanda crescente, tornou-se um dos nós críticos à organização dos serviços de saúde. Entre outros aspectos, os quesitos de acessibilidade, dificuldades de integração entre os componentes da rede, escassos mecanismos de referência e contrarreferência e inconsistente regulamentação tornaram-se os elementos indutores à baixa capacidade de resolubilidade desse componente 32 . 


\section{Figura 5}

Distribuição de respostas para o componente Sistema de Governança. Região de Saúde de Campo Grande, Mato Grosso do Sul, Brasil, $2014-2015$.

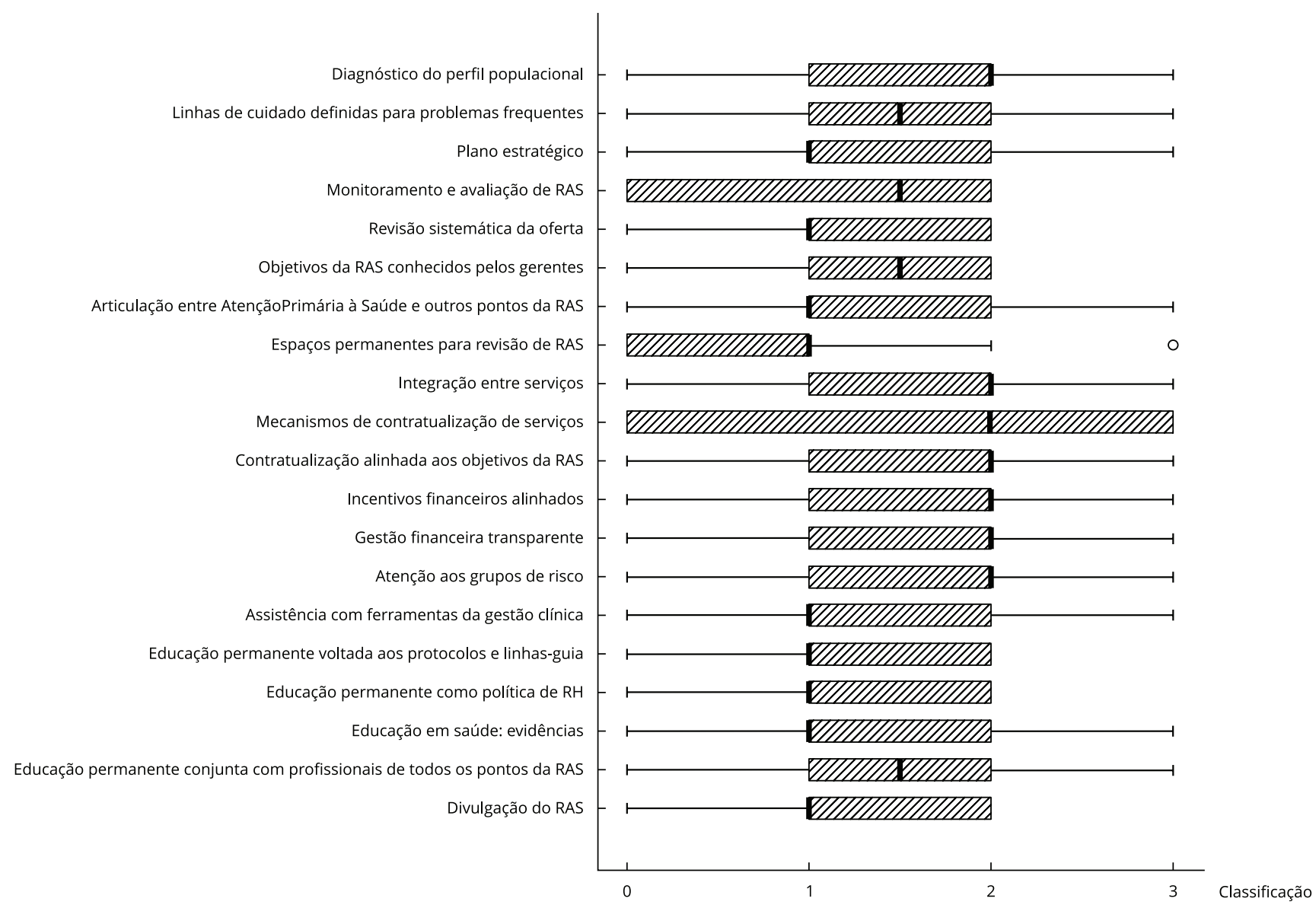

RAS: Rede de Atenção à Saúde; RH: recursos humanos.

Quando analisados os aspectos voltados à integração entre os diferentes pontos e níveis assistenciais, os resultados apresentam-se inexistentes ou incipientes para protocolos e não há integração entre sistemas de informação para visualização dos resultados. Apenas um dos municípios afirma a existência de integração entre os sistemas.

Os Sistemas de Apoio, espaços comuns e transversais a todos os pontos de atenção, compreendem o apoio diagnóstico e terapêutico, sistema de assistência farmacêutica e sistema de informação em saúde 33,34. Cabe aos sistemas de informação permitir o registro de usuários, a coordenação de agendamento e a gestão de dados clínicos. A capacidade de integração das informações clínicas e financeiras é vista como uma importante forma de monitoramento de custo-eficácia e como facilitador do processo de planejamento 35 .

Um sistema de informação ideal promove a agregação dos dados clínicos dos usuários, de modo a auxiliar o processo de planejamento, o reconhecimento de grupos de riscos com necessidades especiais e a gestão do cuidado integral, incluindo a emissão de avisos para a manutenção de vínculo com o usuário 22 . 
Comumente os mecanismos de referência e contrarreferência apresentam fragilidades, especialmente pela incipiente utilização de tecnologias de informação evidenciada neste estudo. Em âmbito internacional, experiências exitosas envolvem a utilização dos sistemas de informação e dos registros eletrônicos em saúde 33 . Os resultados da pesquisa apontam ausência ou insuficiência de sistemas de informação capazes de registrar, acessar e disponibilizar informações clínicas e gerenciais, ou de integrar os diferentes sistemas de informação. Também se verificou que o transporte de material para exame laboratorial precisa ser desenvolvido e é importante para dar segurança clínica e, consequentemente, maior resolubilidade aos serviços, reduzindo o número de encaminhamentos.

Os sistemas logísticos, considerados espaços transversais à RAS, são estabelecidos pelo cartão de identificação dos usuários (cartão SUS), prontuário clínico, sistema de regulação e de transporte em saúde. Os sistemas de transporte em saúde garantem a acessibilidade aos pontos de atenção das RAS. Esses são subdivididos em transporte de usuários (transporte eletivo ou de urgência), de equipes, de material biológico e de resíduos de saúde 33

Foi possível identificar eficientes resultados na emissão do cartão do SUS. Contudo, ainda existem problemas em relação ao prontuário eletrônico, sistema de regulação e sistema de transporte de amostras de exames de análises clínicas, que podem estar associados à complexidade da sua implantação 34 .

O processo regulatório, por meio do Complexo Regulador, começa com base na avaliação dos graus de riscos dos usuários e de normas e protocolos assistenciais e de acesso. Divide-se em três tipologias, a saber: central de regulação de urgência, de internações eletivas, e de consultas e exames 33 . A implantação de centrais de regulação foi umas das medidas adotadas para garantir a integração das redes e o monitoramento das filas de espera pela Atenção Primária à Saúde, mesmo a contrarreferência se demonstrando ainda um pouco tímida. Apesar da implantação de complexos reguladores no estado no ano de 2008, o estudo apontou que ainda há problemas em relação ao sistema de regulação 27 .

Mecanismos de apoio, como a oferta de um adequado transporte sanitário, utilização de ferramentas de tecnologias de informação, atuação territorial e resolutiva, integração entre os pontos de atenção e elaboração, revisão e utilização de protocolos clínicos e de regulação precisam entrar na agenda do processo regulatório 31. Ressalta-se que o aumento do acesso aos serviços de Atenção Primária Saúde vem propiciando a ampliação da demanda às consultas e exames especializados.

A Governança, componente pouco desenvolvido nos achados deste estudo, é um recurso que possibilita o gerenciamento e a integração entre os componentes da rede, e é responsável pelo processo de planejamento, monitoramento, avaliação e tomada de decisão 36,37. A Governança pressupõe a existência de alguns atributos, como eficácia, equidade, eficiência, credibilidade e legitimidade e seus mecanismos efetivos/reais de atuação que devem pautar a operacionalização da assistência 38,39. É um processo interativo de ação coletiva no âmbito das políticas de saúde 37,39.

Seus processos de participação e negociação interinstitucional permitem a gestão de todos os componentes da rede a partir de colegiados regionais (Comissões Intergestores Tripartite - CIT, Bipartite - CIB e Regionais - CIR) com participação social para a promoção da decisão compartilhada. Esse formato de tomada de decisões, no entanto, exige o estabelecimento de mecanismos facilitadores da comunicação entre os atores envolvidos 25,33,40,41.

Resultados semelhantes foram encontrados em outro estudo que apontou a governança incipiente em sistemas fragmentados 36 . Em geral, observa-se que o debate regional ainda é centrado em assuntos classificados como urgentes, com disputas por recursos entre os municípios, além de ser influenciado pela rotatividade dos gestores, grau de autonomia, qualificação técnica e políticas partidárias 41 . A insuficiência de recursos financeiros inviabiliza o cumprimento das ações pactuadas e a execução da proposta de RAS, além de fomentar as desigualdades sociais e regionais 11,41,42. Para favorecer a organização dos serviços em RAS, torna-se iminente a necessidade de ultrapassar a governança meramente política 43 .

A assimilação necessária, pelos tomadores de decisão, de que o adequado funcionamento da RAS é dependente do grau de resolubilidade da Atenção Primária à Saúde, da demanda reprimida, da acessibilidade, de processos de referência e contrarreferência instituídos, da organização dos serviços a partir de linhas de cuidado e previsão de recursos financeiros irá contribuir para a estruturação dos serviços de saúde, superando a fragmentação 25,28,44.

A concepção de governança confere unicidade para todo o sistema de saúde, favorecendo a participação social, com práticas intersetoriais e aproximação com a equidade em saúde. Além disso, o 
processo de ordenação e gestão das redes deve permitir a gestão compartilhada dos sistemas de apoio clínico, administrativo e logístico, bem como força de trabalho suficiente e qualificada para atender a necessidade, integração de sistemas de informação entre os componentes da rede e gerenciamento baseado em resultados, propiciando a melhoria contínua da qualidade. E mais, a alocação de incentivos financeiros deve ser compatível e alinhada com as metas da rede de atenção 22.

São decisões políticas que promovem a superação da gestão fragmentada. Na Alemanha e em outros países da Europa é notável o estímulo e investimento no médico generalista, no intuito de favorecer a continuidade dos cuidados e a condução do sistema de saúde com governança única 45 .

A regulação assistencial deve se basear nas necessidades dos usuários segundo riscos sanitários e sociais, numa lógica de gestão de base populacional, e não com base na oferta de serviços (capacidade instalada), equilibrando o acesso e a concentração de serviços na formulação de políticas de saúde, e com base na lógica da economia de escala e na dimensão geográfica do território.

Este estudo apresenta a limitação de ser baseado em questionários autoaplicáveis, com respostas autorreferidas, sem a busca de evidências.

\section{Conclusões}

Apesar dos avanços ocorridos na saúde pública e no quesito regionalização da saúde, especialmente para atender o cenário de "tripla carga de doenças", sobretudo as doenças crônicas, ainda existem desafios que precisam ser superados para que as RAS se tornem realidade. Os resultados desta pesquisa sugerem que o arcabouço normativo para a instituição das RAS não é, por si só, suficiente para a sua implantação. Acreditamos que tal desvio possa ser atribuído ao modo de "fazer" a gestão do sistema de saúde, nos níveis locais e em suas articulações regionais. É preciso fomentar a construção de planos de ação baseando-se em cenários desejáveis e possíveis, pois agendas tomadas por assuntos imediatos dificilmente contribuirão para superar a fragmentação hoje existente. É necessária a decisão política gestora baseada em informações das necessidades de cada território.

Nesse sentido, o questionário formulado voltado à avaliação ex-ante se mostrou potente para apontar as fragilidades existentes nos municípios e que precisam ser abordadas para que possa haver avanços na formação de redes de atenção, sob a penalidade de comprometer a sua implantação e sua intencionalidade. $\mathrm{O}$ uso do questionário e a análise dos seus resultados permitem a tomada de decisão gestora valendo-se da análise da estrutura e dos processos de trabalho, para qualquer linha de cuidado e território. No caso, o conhecimento da realidade pode ser analisado em conjunto pelos gestores quanto à viabilidade da estratégia selecionada para o enfrentamento das situações, aperfeiçoando a governabilidade.

A gestão, para além dos recursos financeiros e cenários políticos e político-partidários, precisa coordenar tecnicamente os processos de trabalho, definir fluxos e responsabilidades, criar mecanismos de formação contínua e estabelecer formas de aferir o desempenho. Assim, será possível propor e estabelecer redes de atenção e constituir linhas de cuidado para atender as necessidades de saúde das pessoas e a uniformidade nos serviços, considerando todas as especificidades locorregionais.

Dessa maneira, a organização dos serviços em conformidade com os componentes de uma rede de atenção pode anteceder e orientar a sua implantação e, neste sentido, os achados desta pesquisa podem auxiliar na identificação dos limites e potencialidades que devem ser considerados para a conformação da linha de cuidado às pessoas com hipertensão arterial, desde a Atenção Primária à Saúde até os serviços de maior densidade tecnológica, bem como no processo de implantação da Rede de Atenção à Saúde das Pessoas com Doenças Crônicas não só na região de saúde estudada, mas também nas demais regiões de saúde e em outros municípios. 


\section{Colaboradores}

C. M. Santos trabalhou na concepção e desenho da pesquisa, coleta, sistematização e análise das informações, contribuindo na revisão crítica do estudo e elaboração do artigo. A. R. Barbieri contribuiu na concepção do estudo, no recorte metodológico, na revisão crítica e análise dos resultados, e na redação final do artigo e sua aprovação para publicação. C. C. M. Gonçalves colaborou na discussão e análise dos resultados e na redação final do artigo. D. H. Tsuha contribuiu na coleta e sistematização dos dados, na análise estatística, desenvolvimento de figuras e na redação do artigo em sua versão final.

\section{Agradecimentos}

Ao Mestrado Profissional em Saúde da Família da Universidade Federal de Mato Grosso do Sul; Fundação de Apoio ao Desenvolvimento do Ensino, Ciência e Tecnologia do Estado do Mato Grosso do Sul/Conselho Nacional de Desenvolvimento Científico e Tecnológico (FUNDECT/CNPq no 06/2011 - PRONEM) pelo apoio financeiro; secretários de saúde, colaboradores dos municípios e todos que direta ou indiretamente contribuíram para a realização desta pesquisa.

\section{Referências}

1. Chueiri PS, Harzheim E, Gauche H, Vasconcelos LC. Pessoas com doenças crônicas, as redes de atenção e a Atenção Primária à Saúde. Divulg Saúde Debate 2014; 52:114-24.

2. Instituto Brasileiro de Geografia e Estatística. Pesquisa Nacional de Saúde 2013. Percepção do estado de saúde, estilos de vida e doenças crônicas: Brasil, Grandes Regiões e Unidades da Federação. Rio de Janeiro: Instituto Brasileiro de Geografia e Estatística; 2014.
3. Organização Pan-Americana da Saúde. A atenção à saúde coordenada pela APS: construindo as redes de atenção no SUS. Brasília: Organização Pan-Americana da Saúde; 2011.

4. Vargas I, Mogollón-Pérez AS, Unger JP, Silva MRF, Paepe P, Vázquez ML. Regional-based Integrated Healthcare Network policy in Brazil: from formulation to practice. Health Policy Plan 2015; 30:705-17. 
5. Silva SF. Organização de redes regionalizadas e integradas de atenção à saúde: desafios do Sistema Único de Saúde (Brasil). Ciênc Saúde Coletiva 2011; 16:275362.

6. Magalhães Júnior HM. Redes de Atenção à Saúde: rumo à integralidade. Divulg Saúde Debate 2014; 52:15-37.

7. Brasil. Portaria no 4.279, de 30 de dezembro de 2010. Estabelece diretrizes para a organização da Rede de Atenção à Saúde no âmbito do Sistema Único de Saúde (SUS). Diário Oficial da União 2010; $31 \mathrm{dez}$.

8. Brasil. Decreto no 7.508, de 28 de junho de 2011. Regulamenta a Lei no 8.080, de 19 de setembro de 1990, para dispor sobre a organização do Sistema Único de Saúde - SUS, o planejamento da saúde, a assistência à saúde e a articulação interfederativa, e dá outras providências. Diário Oficial da União 2011; 29 jun.

9. Duarte CMR, Pedroso MM, Bellido JG, Moreira RS, Viacava F. Regionalização e desenvolvimento humano: uma proposta de tipologia de Regiões de Saúde no Brasil. Cad Saúde Pública 2015; 31:1163-74.

10. Lima LD, Viana AL, D’Ávila, Machado CV. A regionalização da saúde no Brasil: condicionantes e desafios. In: Scatena JHG, Kehrig RT, Spinelli MAS, organizadores. Regiões de saúde: diversidade e processo de regionalização em Mato Grosso. São Paulo: Editora Hucitec; 2014. p. 21-46.

11. Mendes EV. Comentários sobre as Redes de Atenção à Saúde. Divulg Saúde Debate 2014; 52:38-49.

12. Ribeiro PT. Perspectiva territorial, regionalização e redes: uma abordagem à política de saúde da República Federativa do Brasil. Saúde Soc 2015; 24:403-12.

13. Brousselle A, Champagne A, Contandriopoulos A, Hartz Z. Avaliação: conceitos e métodos. Rio de Janeiro: Editora Fiocruz; 2011.

14. Cohen E, Franco R. Avaliação de projetos sociais. Petrópolis: Editora Vozes; 1993.

15. Champagne CP, Ross RP, Saarela M, Hansen $\mathrm{KF}$, Charalampopoulos D. Recommendations for the viability assessment of probiotics as concentrated cultures and in food matrices. Int J Food Microbiol 2011; 149:185-93.
16. Mendes EV. As redes de atenção à saúde. 2ạ Ed. Brasília: Organização Pan-Americana da Saúde; 2011.

17. Chueiri PS. Proposta de instrumento para a avaliação da coordenação do cuidado e da ordenação das redes de atenção à saúde pela atenção primária no Brasil [Dissertação de Mestrado]. Porto Alegre: Universidade Federal do Rio Grande do Sul; 2013.

18. Rowde P. Essential statistics for the pharmaceutical sciences. Chichester: John Wiley \& Sons; 2007.

19. Ocampo-Rodriguez MV, Betancourt-Urrutia VF, Montoya-Rojas JP, Bautista-Botton DC. Sistemas y modelos de salud, su incidência en las redes integradas de servicios de salud. Revista Gerencia y Políticas de Salud 2013; 12:114-29.

20. Giovanella L. Atenção primária à saúde e coordenação dos cuidados na rede assistencial. Divulg Saúde Debate 2014; 51:30-7.

21. Waibel S, Vargas I, Aller MB, Gusmão R, Henao D, Vázquez ML. The performance of integrated health care networks in continuity of care: a qualitative multiple case study of COPD patients. Int J Integr Care 2015; 15:e029.

22. Pan American Health Organization. Improving chronic illness care through integrated health service delivery networks. Washington DC: Pan American Health Organization; 2012.

23. Lamata Cotanda F. Atención sanitária y rede de servicios. Madrid: Escuela Nacional de Sanidad; 2011

24. Almeida PF, Gérvas J, Freire JM, Giovanella L. Estratégias de integração entre atenção primária à saúde e atenção especializada: paralelos entre Brasil e Espanha. Saúde Debate 2013; 37:400-15.

25. Magalhães Júnior HM, Pinto HA. Atenção Básica enquanto ordenadora da rede e coordenadora do cuidado: ainda uma utopia? Divulg Saúde Debate 2014; 51:14-29.

26. Costa JMBS, Silva MRF, Carvalho EF. Avaliação da implantação da atenção à hipertensão arterial pelas equipes de Saúde da Família do Município do Recife (PE - Brasil). Ciênc Saúde Coletiva 2011; 16:623-33. 
27. Dobashi BF. Tecendo redes na saúde para ampliar o cuidado. Campo Grande: [s.n.]; 2014.

28. Erdmann AL, Andrade SR, Mello ALSF, Drago LC. A atenção secundária em saúde: melhores práticas na rede de serviços. Rev Latinoam Enferm 2013; 21(spe):131-9.

29. Almeida PF, Giovanella L, Mendonça MHM, Escorel S. Desafios à coordenação dos cuidados em saúde: estratégias de integração entre níveis assistenciais em grandes centros urbanos. Cad Saúde Pública 2010; 26:286-98.

30. Almeida PF, Giovanella L, Nunan BA. Coordenação dos cuidados em saúde pela atenção primária à saúde e suas implicações para a satisfação dos usuários. Saúde Debate 2012; 36:375-91.

31. Passos JEF, Rocha LM, Vasconcelos LLC. Estratégia de cuidado na Atenção Especializada Ambulatorial: qualificação e ampliação do acesso. Divulg Saúde Debate 2014; 51:121-8.

32. Conselho Nacional de Secretários de Saúde. Inovação na Atenção Especializada no Brasil. Revista do Conselho Nacional de Secretários de Saúde 2015; ano V, n. 16.

33. Organização Pan-Americana da Saúde. Inovação nos sistemas logísticos: resultados do laboratório de inovação sobre redes integradas de atenção à saúde baseadas na APS. Brasília: Organização Pan-Americana da Saúde/Conselho Nacional de Secretários de Saúde/Conselho Nacional de Secretarias Municipais de Saúde; 2010.

34. Marin HF. Sistemas de informação em saúde: considerações gerais. J Health Inform 2000; 2:20-4.

35. Suter E, Oelke ND, Adair CE, Armitage GD. Ten key principles for successful health systems integration. Healthc Q 2009; 13(Spec No):16-23.

36. Hoffmann EJ, Lima E WB, Barbosa FTRG, Porto HLS, Castro KD, Santana KNO, et al. O funcionamento do sistema governança das redes de atenção à saúde em Monte Claros, Minas Gerais - Brasil. Revista Eletrônica Gestão \& Saúde 2012; 3:788-99.
37. Rodríguez C, Lamothe L, Barten F, Haggerty J. Gobernanza y salud: significado y aplicaciones en América Latina. Rev Salud Pública 2010; 12:151-9.

38. Frenk J, Moon S. Governance challenges in global health. New Engl J Med 2013; 368: 936-42.

39. Hufty M, Báscolo E, Bazzani R. Gobernanza en salud: un aporte conceptual y analítico para la investigación. Cad Saúde Pública 2006; 22 Suppl:S35-45.

40. Granja GF, Zoboli ELCP. Humanização da atenção primária à saúde: gestão em redes e governança local. O Mundo da Saúde 2012; 36:494-501.

41. Santos AM, Giovanella L. Governança regional: estratégias e disputas para a gestão em saúde. Rev Saúde Pública 2014; 48:622-31.

42. Costa LS, Gadelha CAG, Borges TR, Burd P, Maldonado J, Vargas M. A dinâmica inovativa para a reestruturação dos serviços de saúde. Rev Saúde Pública 2012; 46 Suppl 1:76-82.

43. Santos L, Campos GWS. SUS Brasil: a região de saúde como caminho. Saúde Soc 2015; 24:438-46.

44. Barbieri AR, Gonçalves CCM, Cheade MFM, Souza C, Tsuha D, Ferreira KC, et al. Hemodialysis services: are public policies turned to guaranteeing the access? Cad Saúde Pública 2015; 31:1505-16.

45. Rodrigues LBB, Silva PCS, Peruhype R, Palha PF, Popolin MP, Crispim JA, et al. A atenção primária à saúde na coordenação das redes de atenção: uma revisão integrativa. Ciênc Saúde Coletiva 2014; 19:343-52. 


\section{Abstract}

In the context of public health policies, healthcare network is a strategy that aims to promote people's equitable access to services and to reduce fragmentation. The aim of this study was to evaluate the degree of development of components in a healthcare network for hypertension. This was an ex-ante, cross-sectional evaluative study focused on the implementation of a healthcare network for persons with chronic diseases, applying a questionnaire to 17 health administrators from the municipalities (counties) comprising the largest health district in Mato Grosso do Sul State, Brazil. The questionnaire consisted of 65 questions covering the five components: Primary Health Care; Specialized Care; Support Systems; Logistics Systems; and Governance. The study conducted descriptive statistical tests and the classification of services provided in each component using the Friedman test, followed by the Student-NewmanKeuls post hoc test, with significance set at $5 \%$. The results were distributed in quartiles and presented in boxplot graphs. Correlations were established between the dimensions. According to the findings, the components are in an intermediate degree of implementation, with low development of the items needed for establishing networks. Primary Health Care does not coordinate the care, and the Specialized Care and Governance components showed the worst results. The findings indicate predominance of installed services that still fall short of the necessary practices for establishing healthcare networks, which can compromise their implementation.

Health Policy; Health Services; Health Evaluation; Hypertension

\section{Resumen}

En el contexto de las políticas públicas de salud, la red de atención es una estrategia que tiene como objetivo promover la equidad del acceso de las personas a los servicios y reducir su fragmentación. El objetivo fue evaluar el grado de desarrollo de los componentes de una red de atención a la salud para la hipertensión. Se trata de un estudio evaluativo del tipo ex-ante, de corte transversal, dirigido a la implantación de la red de atención a la salud de las personas con enfermedades crónicas con la aplicación de un cuestionario a 17 gestores de salud de los municipios que forman la mayor región de salud de Mato Grosso do Sul, Brasil. El cuestionario está constituido por 65 preguntas que contemplan los cinco componentes: Atención Primaria a la Salud; Atención Especializada; Sistemas de Apoyo; Sistemas Logísticos; y Gobernanza. Se realizaron test estadísticos descriptivos y la clasificación de los servicios prestados en cada componente mediante el test de Friedman, seguido del pos-test de Student-Newman-Keuls, con un nivel de significancia de un 5\%. Los resultados se distribuyeron en cuartiles y presentados en gráficos Boxplot. Se establecieron correlaciones entre las dimensiones. Los resultados apuntan a que los componentes están en el grado intermedio de implantación, con bajo desarrollo en los requisitos necesarios para la constitución de redes. La Atención Primaria a la Salud no coordina el cuidado, y los componentes Atención Especializada y Gobernanza presentaron los peores resultados. Los hallazgos apuntan al predominio de servicios establecidos, incluso distantes de las prácticas necesarias para la composición de redes de atención a la salud, pudiendo comprometer su implantación.

Política de Salud; Servicios de Salud; Evaluación en Salud; Hipertensión
Recebido em 30/Mar/2016

Versão final reapresentada em 18/Jul/2016

Aprovado em 28/Jul/2016 